\title{
Physical parameters of GRB 970508 from its afterglow synchrotron emission
}

\author{
T.J. Galama ${ }^{1}$, R.A.M.J. Wijers ${ }^{2}$, P.M. Vreeswijk ${ }^{1}$, P.J. Groot ${ }^{1}$, J. Van Paradijs ${ }^{1,3}$, C. Kouveliotou ${ }^{4}$, \\ C. Robinson ${ }^{4}$, M. Bremer ${ }^{5}$, R.G. Strom ${ }^{1,6}$, and N. Tanvir ${ }^{7}$ \\ 1 Astronomical Institute "Anton Pannekoek" /CHEAF, Kruislaan 403, 1098 SJ, Amsterdam, The Netherlands \\ 2 Dept. of Physics \& Astronomy, SUNY, Stony Brook, NY, U.S.A. \\ 3 Physics Department, UAH, Huntsville, AL 35899, U.S.A. \\ 4 USRA at NASA/MSFC, Code ES-84, Huntsville AL 35812, U.S.A. \\ 5 Institut de Radio Astronomie Millimétrique, 300 rue de la Piscine, F-38406 Saint-Martin d'Hères, France \\ 6 NFRA, Radio Observatory, Postbus 2, 7990 AA, Dwingeloo, The Netherlands \\ 7 Institute of Astronomy, Madingley Road, Cambridge, UK
}

Received December 29, 1998; accepted May 4, 1999

\begin{abstract}
For the case of an adiabatically expanding blast wave, which is applicable to observed gamma-ray burst (GRB) afterglows at late times, we give expressions to infer the physical properties of the afterglow from the measured spectral features.

We show that enough data exist for GRB 970508 to compute unambiguously the ambient density, $n=0.03 \mathrm{~cm}^{-3}$, and the blast wave energy per unit solid angle, $\mathcal{E}=410^{52} \mathrm{erg} / 4 \pi \mathrm{sr}$. We also compute the energy density in electrons and magnetic field. We find that they are $12 \%$ and $9 \%$, respectively, of the nucleon energy density.
\end{abstract}

Key words: gamma-rays: bursts - radiation mechanisms: non-thermal

\section{Introduction}

The afterglow of the burst of May 8, 1997, was bright for a relatively long time and produced emission from $\gamma$ rays to radio. This enabled a detailed analysis of its broad-band spectrum, confirming in great detail that in the afterglow we see synchrotron emission, and that the dynamical evolution of the expanding blast wave agrees with predictions if the blast wave dynamics are adiabatic (Galama et al. 1998a,b). In principle, one can derive the blast wave properties from the observed synchrotron spectral features. The problem is that the characteristic synchrotron frequencies and fluxes are taken from simple dimensional analysis in the published literature, so they are

Send offprint requests to: T.J. Galama not suitable for detailed data analysis. We amend this situation and correct the coefficients in the equations for the break frequencies by up to a factor 10 . We then use our theoretical results to infer the physical properties of the afterglow of GRB 970508.

\section{Synchrotron radiation from an adiabatic blast wave}

We have derived the synchrotron frequencies and fluxes for a relativistic blast wave expanding in a uniform medium. The results for the peak flux, $F_{\nu_{\mathrm{m}}}$, the synchrotron selfabsorption, $\nu_{\mathrm{a}}$, peak, $\nu_{\mathrm{m}}$, and cooling, $\nu_{\mathrm{c}}$, frequencies are summarised below (details can be found in Wijers \& Galama 1999).

$$
\begin{aligned}
F_{\nu_{\mathrm{m}}}= & 1.15 \frac{h_{70}^{2}}{(\sqrt{1+z}-1)^{2}} \phi_{\mathrm{p}}(1+X) \mathcal{E}_{52} n^{1 / 2} \epsilon_{B}{ }^{1 / 2} \mathrm{mJy} \\
\nu_{\mathrm{a}}= & 2.9710^{8}\left(\frac{p+2}{p+\frac{2}{3}}\right)^{3 / 5} \frac{(p-1)^{8 / 5}}{p-2}(1+X)^{8 / 5} n^{3 / 5} \epsilon_{\mathrm{e}}{ }^{-1} \\
& \epsilon_{B}{ }^{1 / 5} \mathcal{E}_{52}{ }^{1 / 5}(1+z)^{-1} \mathrm{~Hz} \\
\nu_{\mathrm{m}}= & 5.7310^{16} x_{\mathrm{p}}\left(\frac{p-2}{p-1}\right)^{2}(1+X)^{-2} \epsilon_{\mathrm{e}}{ }^{2} \epsilon_{B}{ }^{1 / 2} \mathcal{E}_{52}{ }^{1 / 2} \\
& (1+z)^{1 / 2} t_{\mathrm{d}}{ }^{-3 / 2} \mathrm{~Hz} \\
\nu_{\mathrm{c}}= & 1.1210^{12} \epsilon_{B}{ }^{-3 / 2} \mathcal{E}_{52}{ }^{-1 / 2} n^{-1}(1+z)^{-1 / 2} \\
& t_{\mathrm{d}}{ }^{-1 / 2} \mathrm{~Hz} .
\end{aligned}
$$

Here $x_{\mathrm{p}}$ and $\phi_{\mathrm{p}}$ are the dimensionless spectral location and dimensionless peak flux of a synchrotron spectrum from a power law distribution of electron energies with index $p . X$ is the hydrogen mass fraction, $\epsilon_{\mathrm{e}}$ and $\epsilon_{B}$ are the ratios of 
energy in electrons and energy in the magnetic field to energy in nucleons, respectively, $\mathcal{E}_{52}$ is the blast wave energy per unit solid angle in units of $10^{52} \mathrm{erg}, n$ is the nucleon density, $h_{70}=H_{0} / 70 \mathrm{~km} \mathrm{~s}^{-1} \mathrm{Mpc}^{-1}, z$ the redshift and $t_{\mathrm{d}}$ the time in days since trigger (see Wijers \& Galama 1999). We have adopted an $\Omega=1, \Lambda=0$ universe.

\section{The inverse solution}

Equations (1)-(4) are four independent relations among the four parameters of interest $\mathcal{E}_{52}, n, \epsilon_{\mathrm{e}}$, and $\epsilon_{B}$. This means we can solve for all parameters of interest if we have measured all three break frequencies (not necessarily at the same time) and the peak flux of the afterglow. In addition this requires us to know the redshift of the burst, the electron index $p$, and the composition parameter, $X$, of the ambient medium. We define the constants $C_{\mathrm{a}} \equiv \nu_{\mathrm{a}} / \nu_{\mathrm{a} *}, C_{\mathrm{m}} \equiv \nu_{\mathrm{m}} t_{\mathrm{dm}}^{3 / 2} / \nu_{\mathrm{m} *}, C_{\mathrm{c}} \equiv \nu_{\mathrm{c}} t_{\mathrm{dc}}^{1 / 2} / \nu_{\mathrm{c} *}$, and $C_{\mathrm{F}}=F_{\nu_{\mathrm{m}}} / F_{\nu_{\mathrm{m}} *}$. Here starred symbols denote the numerical coefficients in each of the four equations, and times $t_{\mathrm{dm}}, t_{\mathrm{dc}}$ denote the time at which the quantity in question was measured. Rearranging the four equations then yields

$$
\begin{aligned}
\mathcal{E}_{52}= & C_{\mathrm{a}}{ }^{-\frac{5}{6}} C_{\mathrm{m}}{ }^{-\frac{5}{12}} C_{\mathrm{c}}{ }^{\frac{1}{4}} C_{\mathrm{F}}{ }^{\frac{3}{2}} x_{\mathrm{p}}^{\frac{5}{12}} \phi_{\mathrm{p}}^{-\frac{3}{2}}(p-1)^{\frac{1}{2}}\left(\frac{p+2}{p+\frac{2}{3}}\right)^{\frac{1}{2}} \\
& (1+X)^{-1}(1+z)^{-\frac{1}{2}}\left(\frac{\sqrt{1+z}-1}{h_{70}}\right)^{3} \\
\epsilon_{\mathrm{e}}= & C_{\mathrm{a}^{\frac{5}{6}}} C_{\mathrm{m}}{ }^{\frac{11}{12}} C_{\mathrm{c}}{ }^{\frac{1}{4}} C_{\mathrm{F}}{ }^{-\frac{1}{2}} x_{\mathrm{p}}^{-\frac{11}{12}} \phi_{\mathrm{p}}^{\frac{1}{2}} \frac{(p-1) \frac{1}{2}}{p-2}\left(\frac{p+2}{p+\frac{2}{3}}\right)^{-\frac{1}{2}} \\
& (1+X)(1+z)^{\frac{1}{2}}\left(\frac{\sqrt{1+z}-1}{h_{70}}\right)^{-1} \\
\epsilon_{B}= & C_{\mathrm{a}}{ }^{-\frac{5}{2}} C_{\mathrm{m}}{ }^{-\frac{5}{4}} C_{\mathrm{c}}{ }^{-\frac{5}{4}} C_{\mathrm{F}}{ }^{\frac{1}{2}} x_{\mathrm{p}}^{\frac{5}{4}} \phi_{\mathrm{p}}^{-\frac{1}{2}}(p-1)^{\frac{3}{2}}\left(\frac{p+2}{p+\frac{2}{3}}\right)^{\frac{3}{2}} \\
& (1+X)(1+z)^{-\frac{5}{2}}\left(\frac{\sqrt{1+z}-1}{h_{70}}\right)^{-\frac{5}{2}} \\
n= & C_{\mathrm{a}}{ }^{\frac{25}{6}} C_{\mathrm{m}}{ }^{\frac{25}{12}} C_{\mathrm{c}}{ }^{\frac{3}{4}} C_{\mathrm{F}}{ }^{-\frac{3}{2}} x_{\mathrm{p}}^{-\frac{25}{12}} \phi_{\mathrm{p}}^{\frac{3}{2}}(p-1)^{-\frac{5}{2}}\left(\frac{p+2}{p+\frac{2}{3}}\right)^{-\frac{5}{2}} \\
& (1+X)^{-1}(1+z)^{\frac{7}{2}}\left(\frac{\sqrt{1+z}-1}{h_{70}}\right)^{-3} .
\end{aligned}
$$

\section{Physical parameters of GRB 970508}

From the light curve behaviour and broad-band spectrum of GRB 970508, we deduced the properties of the burst required to calculate the physical parameters of the afterglow; at $t=12.1 \mathrm{~d}$ after trigger, the break frequencies are $\nu_{\mathrm{a}}=2.510^{9} \mathrm{~Hz}, \nu_{\mathrm{m}}=8.610^{10} \mathrm{~Hz}$, and $\nu_{\mathrm{c}}=1.610^{14} \mathrm{~Hz}$ (Galama et al. 1998a). The peak flux is $F_{\nu_{\mathrm{m}}}=1.7 \mathrm{mJy}$ and the electron index $p=2.2$. After the first $500 \mathrm{~s}$ electrons no longer cooled efficiently and the afterglow must evolve adiabatically. We use a redshift of $z=0.835$ for GRB 970508 (Bloom et al. 1998) and $H_{0}=70 \mathrm{~km} \mathrm{~s}^{-1} \mathrm{Mpc}^{-1}$. Finally, we adopt $X=0.7$ for the

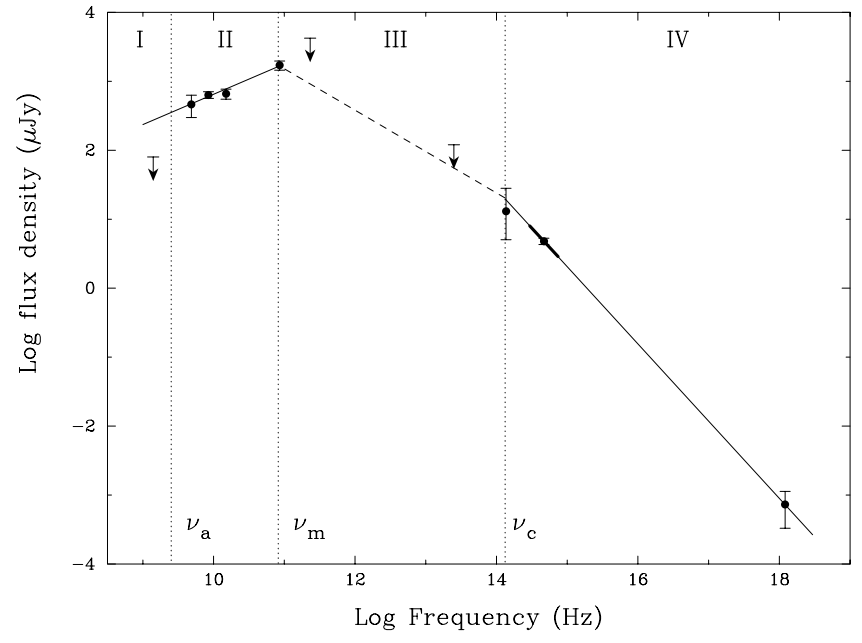

Fig. 1. The X-ray to radio spectrum of GRB 970508 on May 21.0 UT (12.1 days after the event) from Galama et al. (1998a). Indicated are the inferred values of the break frequencies $\nu_{\mathrm{a}}$, $\nu_{\mathrm{m}}$ and $\nu_{\mathrm{c}}$ for May 21.0 UT

composition of the ambient medium. Using further that $x_{2.2}=0.580, \phi_{2.2}=0.611$, we find

$\mathcal{E}_{52}=3.5 \quad n=0.03 \mathrm{~cm}^{-3}$

$\epsilon_{\mathrm{e}}=0.12 \epsilon_{B}=0.09$.

\section{Discussion}

We have demonstrated for the first time that the electron and magnetic field energy densities are close to but somewhat below equipartition value. The ambient density is on the low side of normal for a disc of a galaxy but definitely higher than expected for a halo, lending support to the notion that bursts occur in gas-rich environments.

It is interesting to compare the properties at late times with those derived from radio observations. The scintillation size after 1 month is about $10^{17} \mathrm{~cm}$ (Frail et al. 1997), whereas our formulae give a transverse size of $310^{17} \mathrm{~cm}$, in reasonable agreement given the statistical nature of the scintillation size and our neglect of the gradients in properties in the transverse direction. The Lorentz factor at this time is 3 , so the evolution is still in the relativistic regime. The co-moving field at this time is $B^{\prime}=0.06 \mathrm{G}$.

\section{References}

Bloom J.S., et al., 1998, ApJ 507, L25

Frail D., et al., 1997, Nat 389, 261

Galama, et al., 1998a, ApJ 500, L97

Galama, et al., 1998b, ApJ 500, L101

Wijers R.A.M.J., Galama T.J., 1999, ApJ (in press), Sept. 20 issue, preprint astro-ph/9805341 